\title{
Politique
}

\section{Les femmes et la consommation collective : les enjeux de l'engagement politique}

\section{Caroline Andrew}

Numéro 5, hiver 1984

Femmes et pouvoir

URI : https://id.erudit.org/iderudit/040447ar

DOI : https://doi.org/10.7202/040447ar

Aller au sommaire du numéro

Éditeur(s)

Société québécoise de science politique

ISSN

0711-608X (imprimé)

1918-6584 (numérique)

Découvrir la revue

Citer cet article

Andrew, C. (1984). Les femmes et la consommation collective : les enjeux de

l'engagement politique. Politique, (5), 109-122. https://doi.org/10.7202/040447ar d'utilisation que vous pouvez consulter en ligne.

https://apropos.erudit.org/fr/usagers/politique-dutilisation/ 


\title{
Les femmes et la consommation collective: les enjeux de l'engagement politique
}

\author{
Caroline Andrew \\ Université d'Ottawa
}

Où sont les femmes en politique? Voilà une question importante pour toutes celles et tous ceux qui réfléchissent sur la place des femmes dans nos sociétés modernes. Nous savons que la présence des femmes est minime à tous les échelons et à toutes les instances de la vie politique mais il est important d'aller plus loin que cette première constatation et de regarder les différences qui existent dans la représentation politique des femmes entre différentes sphères de la scène politique. C'est en examinant ces différences, et surtout en tentant de les expliquer, que nous pourrons mieux comprendre les mécanismes de l'engagement des femmes en politique.

La thèse de cet article est que l'implication politique des femmes est liée à des enjeux de consommation collective. C'est donc en approfondissant notre compréhension de la gestion des enjeux de consommation collective dans la société québécoise que nous pourrons comprendre où se trouvent les femmes en politique et pourquoi.

Afin de démontrer notre thèse, nous allons commencer en tentant de chiffrer la présence actuelle des femmes à certaines instances de la vie politique québécoise. Ensuite nous allons passer en revue un certain nombre d'hypothèses qui tentent d'expliquer 
les différences constatées dans la présence des femmes dans les lieux de prise de décision collective. Ce survol des explications nous amènera à la question de la consommation collective. Nous terminerons, finalement, avec une discussion des conséquences pratiques qui pourraient découler de notre argument théorique au sujet des liens entre la politique, les femmes et les enjeux de consommation collective.

Comme nous l'avons constaté au début de cet article, la présence des femmes est faible partout sur la scène publique au Québec. L'Assemblée nationale du Québec compte maintenant, depuis les élections partielles du 5 décembre 1983, 10 femmes sur 122 députés $(8,1 \%)^{1}$. Au niveau fédéral à la Chambre des Communes, la députation québécoise inclut 6 femmes sur 75 , ou $8 \%^{2}$. Sur le plan municipal, le pourcentage des femmes augmente mais il reste très limité. En 1981, 7,5\% des conseillers municipaux au Québec ont été des femmes quoiqu'en 1980 le pourcentage ne s'élevait qu'à $4,1 \%{ }^{3}$. Mais ces paliers ne sont pas les seuls lieux de prise de décision collective et, en élargissant la vision classique de la scène politique, nous pouvons inclure les commissions scolaires et même les conseils des centres locaux de services communautaires (CLSC) ${ }^{4}$. Et ici, effectivement, même si la représentation des femmes est loin d'être égale à leur proportion

1. Lise Bacon, Madeleine Bélanger, Joan Dougherty, Louise Harel, Carmen Juneau, Hughette Lachapelle, Thérèse Lavoie-Roux, Denise Leblanc-Bantey, Pauline Marois, Aline St-Amand.

2. Suzanne Beauchamp-Niquet, Monique Bégin, Eva Côté, Céline HervieuxPayette, Thérèse Killens, Jeanne Sauvé. Avec la nomination de Jeanne Sauvé comme gouverneur-général, le total va peut-être être réduit à 5 .

3. Nicole Campeau, "Prendre le pouvoir ... municipal», La Gazette des femmes. 4-3 septembre 82,9 .

4. Nous avons inclus les CLSC, même si seulement une partie des membres des conseils sont élus, à cause de l'importance des mécanismes de représentation mis en place. Sur la création et le fonctionnement des CLSC, voir Frédéric Lesemann, Du pain et des services, Montréal, Éditions Saint-Martin, 1981; Jacques Godbout, La participation contre la démocratie, Montréal, Éditions Saint-Martin, 1983. 
dans la population, nous retrouvons une présence plus importante des femmes. Par exemple, 23,7\% des candidats élus dans les élections scolaires de juin 1980 était des femmes ${ }^{5}$. Et, au niveau des CLSC, $18,9 \%$ des présidents de conseils des CLSC (14 sur $74)^{6}$, était des femmes en 1982. Il y a donc des femmes au Québec qui occupent des postes sur la scène publique. D'où vient cet engagement et, surtout, comment expliquer la présence des femmes dans certains secteurs plutôt que dans d'autres.

Il y a toute une série de facteurs qui peuvent expliquer les différences que nous avons constatées dans la représentation des femmes à différentes instances de l'État. Une première piste que nous retrouvons dans certaines études explique cette différence par l'importance et la non-importance de différents niveaux de la scène politique ${ }^{7}$. Les femmes s'y retrouvent, selon cette explication, dans les secteurs de l'éducation et des affaires sociales parce que ces secteurs sont des secteurs politiques moins importants, clairement dominés par les autres gouvernements en termes de pouvoir, de prestige, d'autonomie et de responsabilité. Il est clair que les commissions scolaires perdent de plus en plus d'importance et d'autonomie, devenant pour ainsi dire les agents (ou les prisonniers) des gouvernements provinciaux. Quant aux CLSC, ils ont toujours été soumis au Ministère des affaires sociales. Cette perte de statut s'accompagnera donc d'une féminisation dans le personnel politique; la marginalisation et la féminisation allant de pair. Tout comme la médecine ou le droit se féminisent en même temps que ces professions perdent leur prestige, on peut interpréter la présence

5. Evelyne Tardy et al., La politique: un monde d'hommes?, Montréal, Hurtubise HMH, 1982, 6.

6. Chiffres calculés à partir du Répertoire des établissements de santé et de services sociaux, Gouvernement du Québec, Ministère des affaires sociales, 1982.

7. M. Janine Brodie et Jill McCalla Vickers, Canadian Women in Politics: An Overview, Institut canadien de recherches pour l'avancement de la femme, Ottawa, 1982, 15-16. 
des femmes aux paliers scolaire et des affaires sociales de la même façon.

Cette interprétation se complète en référant aux théories sur la socialisation des femmes, socialisation à la passivité et à l'acceptation des rôles inférieurs. «La crainte de la réussite» oriente les femmes vers les secteurs de moindre importance et ceci, dans la sphère politique, les amène vers les commissions scolaires et les conseils sociaux. Les hommes qui s'intéressent à la politique aspirent à jouer des rôles sur les grandes scènes politiques, les femmes se contentent de moins.

Le deuxième type d'explication pour la présence plus forte des femmes dans certains secteurs de la vie politique est en fonction des forces politiques qui dominent ou contrôlent ces différentes scènes politiques. Particulièrement, il faut s'interroger sur le rôle des partis politiques dans la représentation des femmes. Si nous considérons que les partis agissent comme un frein à la participation des femmes à la vie politique, leur absence, par exemple au palier scolaire, pourrait expliquer le fait que les femmes sont plus nombreuses là où les partis sont inexistants. Des études, particulièrement celles de M. Janine Brodie et Jill McCalla Vickers ${ }^{8}$, suggèrent que les partis limitent les candidatures féminines, soit en les écartant complètement, soit en les reléguant à des circonscriptions perdues d'avance.

Mais, si l'absence des partis favorise les femmes, comment expliquer qu'il n'y ait pas plus de candidates et d'élues au plan municipal. Les partis politiques sont absents ou, du moins, les partis politiques qui se retrouvent à d'autres niveaux ne participent pas de façon officielle aux élections municipales. Pour comprendre ce phénomène, il faut d'abord se rappeler que la plupart des

8. Voir surtout M. Janine Brodie et Jill McCalla Vickers, «Gates and Gate keepers and Women Legislative Candidates», Communication présentée à la réunion annuelle de l'Association canadienne de science politique, 1978. 
grandes villes au Québec ont des sortes de partis politiques au niveau municipal et donc que notre argument du rôle des partis comme frein à la représentation des femmes peut tenir. À Montréal notamment, le Parti civique joue un rôle très important dans la sélection des candidats et le nombre des femmes choisies est très limitée.

L'absence des partis simplifie la représentation politique des femmes. Au palier scolaire, il y a très peu de barrières entre la volonté personnelle et la candidature comme telle. À part la candidate elle-même, les seuls agents de sélection sont les électeurs. Aux niveaux québécois ou fédéral, les partis jouent un premier rôle de sélection, un rôle qui n'a guère facilité les candidatures féminines. Selon cette interprétation ce sont les partis politiques qui empêchent les femmes d'être présentes.

Il est possible que cette situation change, notamment avec un changement dans le mode de scrutin. Il est clair, par exemple, qu'une des conséquences envisagées du système de la représentation proportionnelle régionale modérée est un nombre accru de candidates. Si le scrutin se fait sur une base régionale, il serait impensable, croit-on, de présenter seulement des candidats. Les partis seront poussés à s'ouvrir aux femmes et loin de freiner leurs candidatures, les partis pourraient même se mettre à rechercher spécialement les candidates. mais, la réforme électorale n'est pas encore faite au Québec et notre analyse du rôle des partis vaut, selon nous, pour la situation actuelle.

Le troisième type d'explication aborde cette question du point de vue des femmes, par le biais d'une analyse de leur vie quotidienne plutôt que par celui d'une analyse de leur socialisation, comme dans la première interprétation. Les femmes se retrouvent plus en politique scolaire ou en politique d'affaires sociales car

9. Trois femmes (Carmen Millette, Nicole Gagnon-Larocque, Jocelyne Ménard) siègent au conseil dont deux du Parti civique. Sur les 58 membres du conseil, 40 sont du Parti civique. 
c'est l'activité politique la plus accessible pour elles. La très grande majorité des femmes demeurent responsables des relations familiales, de l'éducation des enfants et des tâches domestiques. Il est donc difficile pour elles de viser des carrières politiques qui les éloigneront de leurs familles. Ou, plus exactement, nous devrions dire qu'il est encore très difficile pour les femmes de poursuivre des carrières politiques et infiniment plus difficile quand ces carrières politiques impliquent un travail à plein temps ou des déplacements géographiques importants.

L'entrée massive des femmes sur le marché du travail n'a guère changé cette situation; voire l'a même aggravée. Les études démontrent en effet que la participation des femmes au marché du travail n'a pas été compensée par une participation égale des hommes aux tâches domestiques ${ }^{10}$. Les femmes sont aux prises avec une double vie de travail; le travail rémunéré se conjugue avec la responsabilité pour l'éducation des enfants et pour l'organisation de la vie familiale.

Toute activité politique d'une certaine intensité, à quelque niveau que ce soit, implique des réunions et des obligations qui ne sont pas facilement conciliables avec une vie de famille. Mais quand cette activité politique implique des périodes régulières en dehors du lieu de résidence, les contradictions entre le rôle de mère/conjointe et celui d'acteur politique grandissent. Ghislaine Chénier, seule femme élue dans la ville de Hull, décrit cette réalité.

...les comités d'hommes par exemple sont à cinq heures, les réunions, les heures de bureau, tout est en fonction des hommes! Mais quand il y aura plus de femmes au conseil, des comités à cinq heures, il n'y en aura plus"'.

10. Pat Armstrong et Hugh Armstrong, The Double Ghetto, Toronto, McClelland and Stewart, 1978, 64-66.

11. Antonio Almeida, Yvan M. Dery, Sylvie M. Trépanier, Ghislaine Chénier Conseillère municipale, Travail présenté à Denyse Côté, Département de science politique, University d'Ottawa, 1983, Annexe, 17-18. 
Dans un tel contexte, les avantages d'une activité politique que l'on peut faire à temps partiel sont réels. Les postes, au niveau de l'Assemblée nationale ou de la Chambre de Communes sont de plus en plus des postes à plein temps et même la carrière politique au niveau des grandes villes va dans le même sens. Mais les obligations d'un poste élu au niveau scolaire ou les obligations d'un poste au niveau d'un conseil de CLSC n'imposent pas un choix entre les responsabilités familiales et les responsabilités publiques - on peut être commissaire scolaire et mère de famille en même temps.

Cette interprétation suggère que les contraintes quotidiennes jouent un rôle très important dans la détermination de nos activités. Ce sont moins les questions d'idéologies ou des choix rationnels qui nous orientent, c'est l'organisation quotidienne de nos vies qui facilite certaines options et en bloque d'autres. Les femmes optent davantage pour la politique scolaire et la politique d'affaires sociales parce qu'elles permettent la combinaison de leurs activités familiales et de leurs intérêts politiques et ce, pour des raisons de temps et d'espace.

Cette explication en fonction de l'organisation quotidienne de la vie est complémentaire de l'interprétation suivante, celle découlant de l'étude des canaux de participation et, dans le cas des femmes, du lien entre le bénévolat et l'action politique. Janine Brodie a très clairement démontré dans sa thèse Pathways to Public Office: Canadian Women in the Post-War Years l'importance du bénévolat pour les candidates et surtout pour celles qui se présentent aux élections municipales. Les femmes qui se présentent aux élections fédérales ou provinciales ont plus généralement œuvré dans des partis politiques tandis que les candidates au plan municipal sont souvent passées des activités bénévoles à l'action politique.

This chapter suggests that voluntary groups do indeed play an important role as political apprenticeship structures for women, particularly for those seeking municipal office. ... Group activity instructs the political careers of many women politicians but group-induced political learning was most important for women without 
political party experience. Moreover, those politicized by groups disproportionately pursue election at the municipal level of government ${ }^{12}$.

Le bénévolat est donc très souvent l'expérience qui porte les femmes vers la politique et le bénévolat des femmes se fait beaucoup dans les secteurs d'éducation, d'affaires sociales et de santé. Dans l'étude sur les mairesses au Québec, les auteures constatent que "pour les femmes c'est davantage le fait d'œuvrer et d'être connues par le biais des organisations locales qui paraît le plus déterminant pour être sollicitées par les citoyens»" ${ }^{13}$, tandis que "pour les hommes, il semble bien que ce soit leur expérience politique antérieure au conseil municipal ou ailleurs qui les aide à arriver au poste de maire» ${ }^{14}$. Ce même phénomène est illustré par l'entrevue avec Ghislaine Chénier, conseillère au conseil municipal de Hull. À la question «Avant d'occuper la fonction de conseillère municipale, aviez-vous un emploi quelconque à plein temps?», elle a surtout insisté sur son travail bénévole, indiquant que, pour elle, la préparation pour la politique était le bénévolat.

Non, je travaillais à temps partiel, les avant-midi. J'ai toujours été très active. Je m'occupais de comité d'école des parents... Je me suis occupée de l'école dans le comité des parents et puis j'ai été bénévole au centre Mechtilde pour femmes en détresse ${ }^{15}$.

Cette citation est intéressante car elle nous décrit le passage d'un rôle traditionnel de mère à celui de bénévole et ensuite de militante politique. C'était d'abord l'éducation de ses enfants, ensuite les questions d'éducation plus générale et finalement l'activité politique encore plus générale. Les postes occupés bénévolement par les femmes touchent surtout les sphères traditionnelles des

12. M. Janine Brodie, Patbways to Public Office: Canadian Women in the post-war years, Thèse de doctorat, Université Carleton, 1981, 132-4.

13. Evelyne Tardy, et al., La politique: un monde d'bommes?, Montréal, Hurtubise $\mathrm{HMH}, 1982,44$.

14. Ibid.

15. Almeida, Dery, Trépanier, op. cit., Annexe, 1. 
femmes - éducation, santé, bien-être, - la reproduction de la force de travail. Ces activités les acheminent vers la politique.

Selon cette interprétation le bénévolat joue plusieurs rôles. Dans bien des cas, les expériences bénévoles permettent aux femmes de développer leurs capacités d'organisation. Cette possibilité est donnée plus souvent aux hommes par leur participation au marché du travail. Parce que les femmes travaillent moins souvent en dehors du foyer et parce que les types d'emplois qu'elles occupent sur le marché du travail les conduisent rarement à des postes où il est possible de découvrir et de développer leurs talents d'organisation et de leadership, elles ont peu de possibilité, à travers leur vie professionnelle, de se préparer pour une carrière politique. Grâce au bénévolat, des femmes ont découvert l'importance des questions d'organisation collective et ont développé leurs capacités à traiter de ces questions avec efficacité. De plus, ces expériences ont mis les participantes en contact avec la politique et avec les dirigeants politiques. Ces contacts ont permis à un certain nombre de femmes de se mesurer aux élus et de se rendre compte qu'elles pourraient faire mieux.

Finalement nous arrivons à notre dernière interprétation, celle qui cherche une cohérence entre le contenu des politiques et la participation. Basée sur une vision des acteurs rationnels, cette interprétation cherche à expliquer la participation des femmes à certains secteurs politiques par le contenu des politiques mises en œuvre dans ces secteurs. Certaines études récentes établissent des liens entre les activités visant la reproduction de la force de travail, ou les activités de consommation collective, et les femmes ${ }^{16}$.

Les tâches traditionnelles des femmes - le travail ménager, l'éducation des enfants - démontrent le rapport entre la reproduction de la force de travail et les femmes. Elles se sont toujours

16. Cynthia Cockburn, The Local State: Management of Cities and People, London, Pluto Press, 1978; Elizabeth Wilson, Women and the Welfare State, London, Tavistock, 1977. 
occupées de la reproduction de la force de travail, en faisant le ménage pour les travailleurs actuels et en élevant des travailleurs futurs. La sphère de la consommation est la sphère des femmes et les questions de consommation collective et de consommation individuelle sont intimement liées. Les femmes sont donc les personnes qui connaissent mieux les enjeux de la reproduction de la force de travail et celles qui sont les plus touchées par ces questions. Il est donc logique que leur participation politique concerne davantage les secteurs de l'État qui s'occupent particulièrement de ces questions. Au Québec, et c'est ce que nos chiffres ont confirmé, les femmes s'engagent surtout dans les réseaux d'éducation et d'affaires sociales.

En suggérant un certain lien entre les questions de consommation collective et la présence des femmes, cette interprétation recoupe plusieurs dimensions des autres explications présentées. L'implication politique spécifique des fernmes se fait à cause de la spécialisation des rôles des femmes dans le domaine de la consommation. Ce rapport se noue par la participation des femmes dans les activités bénévoles, activités qui amènent un certain nombre de femmes à passer du secteur bénévole au secteur politique. L'entrée sur la scène scolaire est plus facile, étant donné l'absence des partis politiques qui, à d'autres niveaux d'activité politique, agissent comme des barrières à la participation des femmes. Ce passage des intérêts «traditionnels» des femmes, via le bénévolat, à la politique se fait également plus aisément au niveau scolaire ou dans le réseau d'affaires sociales pour les raisons de proximité géographique et de flexibilité d'horaire. Vie de famille et vie politique sont moins difficiles à combiner quand les obligations politiques sont à temps partiel et dans la même ville que la vie de famille. Dans cet enchaînement de facteurs le lien entre consommation collective, activité politique et femmes nous semble particulièrement important. 
Pourquoi ce lien entre le rôle politique des femmes et les enjeux de consommation collective ne s'est-il pas plus traduit dans les chiffres pour la représentation politique des femmes par palier de gouvernement? Plus spécifiquement, pourquoi le lien constaté ailleurs entre femmes et politique municipale ne s'est-il pas vérifié au Québec ${ }^{17}$ ? C'est le cas notamment en Grande-Bretagne où, en 1976, 17\% des conseillers locaux étaient des femmes, comparé à seulement $4 \%$ des élus au niveau national ${ }^{18}$. En GrandeBretagne c'est le palier local qui s'occupe très clairement des questions de consommation collective et c'est le palier local qui a la plus forte représentation politique des femmes.

Depuis Manuel Castells la définition de la politique urbaine en fonction des questions de consommation collective ou de reproduction de la force de travail est courante et, bien que Castells ait distingué politique urbaine et politique municipale, lui et d'autres ont démontré l'importance des questions de consommation collective pour la politique municipale ${ }^{19}$. Une étude récente de la situation européenne insiste sur l'importance des enjeux de consommation pour définir l'État local ${ }^{20}$.

Mais, au Québec, qu'est-ce qui se passe? Est-ce que la politique municipale s'occupe des questions de consommation

17. Dans une première version de cet article j'avais été amenée par les expériences d'ailleurs à affirmer un lien entre le palier municipal et les femmes au Québec. Mes remerciements aux lecteurs pour m'avoir rappellé le sens des chiffres, rappel d'autant plus utile que cela m'a permis de revenir sur l'interprétation de la politique municipale au Québec en termes de la consommation collective. Voir également la note 22.

18. Stephen L. Bristow, «Women Councillors - an Explanation of the Underrepresentation of Women in Local Government »L, Local Government Studies, mai/juin 1980, 75.

19. Manuel Castells, «Crise de l'État, consommation collective et contradictions urbaines» dans Nicos Poulantzas, La crise de l'État, Paris, Presses universitaires de France, 1976; Manuel Castells et al., Crise du logement et mouvements sociaux urbains, Paris, Mouton, 1978.

20. Patrick Dunleavy, "The Limits of Local Government», Groupe de recherche ANCAN, 1983, 25. "The key concerns of local government in the dual state thesis are social consumption ones». Dunleavy constate que c'est le cas britannique qui correspond le plus à cette thèse mais qu'en général, elle se vérifie pour l'ensemble des pays européens. 
Dépenses brutes générales des gouvernements locaux au Québec, 1978²1

\begin{tabular}{|c|c|c|}
\hline & $\$$ (million) & $\%$ \\
\hline Éducation & 3026.9 & 47,4 \\
\hline Transport et voirie & 712.1 & 11,2 \\
\hline Eaux, ordures ménagères & 585.3 & 9,2 \\
\hline Protection & 470.6 & 7,4 \\
\hline Loisirs & 320.4 & 5,0 \\
\hline Total & 6382.1 & \\
\hline
\end{tabular}

collective? Au Québec, ni la santé, ni le bien-être social ne relèvent du palier local; quant à l'éducation, tout en ayant des assises locales, elle n'est pas du ressort des municipalités. Les commissions scolaires sont élues directement et les taxes scolaires sont prélevées directement par celles-ci. L'éducation est de loin le secteur le plus important des dépenses locales mais elles échappent au pouvoir municipal. Pour mieux situer l'activité municipale, le tableau suivant indique les catégories les plus importantes des dépenses totales des gouvernements locaux au Québec en 1978.

Le tableau n'appuie pas la thèse de la définition de la politique municipale en termes de consommation collective. Les transports, les aqueducs, la police et les pompiers - ce sont tous des domaines qui sont autant, sinon plus, liés au secteur de la production qu'à celui de la consommation. À lire ce tableau, les gouvernements municipaux ne sont pas particulièrement liés aux fonctions de consommation collective mais plutôt a un rôle d'appui à l'accumulation et à l'organisation de la production ${ }^{22}$.

21. Provincial and Municipal Finances 1981, Association canadienne d'études fiscales, Toronto, 1981, 53. Nous n'avons pas inclus les secteurs de l'administration générale ni le service de la dette.

22. Cette même ré-interprétation a été proposée par Warren Magnusson dans «Urban Politics and the Local State», 1983. Magnusson suggère que les analystes ont trop facilement emprunté la définition européenne de la politique municipale en termes des enjeux de consommation collective et qu'ici les fonctions liées à la production sont restées importantes au palier local. 
Tout en acceptant cette conclusion, il faut la pondérer quelque peu. D'abord, les transports et les aqueducs servent aussi le secteur résidentiel et donc ils peuvent être considérés comme relevant partiellement de la consommation. De plus, il est possible de dire que certains domaines liés à la consommation deviennent plus importants au niveau municipal. Les loisirs $(1,8 \%$ des dépenses locales en 1945 et 5,0\% en $1978^{23}$ ) en sont un exemple.

Nous savons, aussi, que le domaine municipal au Québec se transforme actuellement sous l'impulsion de la régionalisation ${ }^{24}$. Aux origines du débat sur la régionalisation le gouvernement québécois a voulu créer de véritables gouvernements régionaux, ayant des pouvoirs dans les domaines de consommation collective, comme l'éducation, les affaires sociales, etc. Les municipalités régionales de comté (MRC) telles que créés n'ont pas ces pouvoirs étendus, elles n'ont qu'une responsabilité dans le domaine de l'aménagement. Mais nous ne savons pas encore comment va se faire l'évolution des pouvoirs des MRC et certainement les analystes ont beaucoup parlé de la possibilité de leur donner d'autres responsabilités. Par exemple, toujours dans le domaine de la consommation collective, la possibilité d'une intégration du réseau des affaires sociales, notamment les CLSC, aux MRC a été envisagée. Les CLSC au lieu d'être gérés par des conseils spécifiquement nommés et élus pour ce seul but, seraient gérés par les conseils régionaux. De cette façon, les politiques dans les domaines de la santé publique, des garderies, d'organisation communautaire, etc.

23. Les chiffres pour 1945 sont pris dans Louise Quesnel-Ouellet, Étude des fonctions du gouvernement local, Groupe de recherche ANCAN, 1983, 35 et ceux pour 1978, de Provincial and Municipal Finances 1981. op. cit.

24. Sur la régionalisation, voir $\mathrm{Rita} B$ issonette, La régionalisation municipale au Québec 1960-1980, Thèse de maîtrise, Département de science politique, Université d'Ottawa, 1982; Jacques Léveillée, L'aménagement du territoire au Québec, Montréal, Nouvelle optique, 1982; Jacques Léveillé et Marie-Odile Trépanier, «Évolution de la législation relative à l'espace urbain au Québec", Droit et Société urbaine au Québec, la Revue juridique Thémis, $16-1 / 2,1981-2$. 
feront partie des responsabilités régionales. Les conseils régionaux seront forcément beaucoup plus impliqués dans les questions de consommation collective.

Comment vérifier notre thèse? Comment voir si la question des enjeux touchant la consommation collective est véritablement un canal important de passage des activités traditionnelles des femmes vers la prise en charge d'un rôle public accru. Une piste de réponse est suggérée par nos données - nous pouvons modifier les responsabilités municipales et régionales de façon à inclure plus de secteurs relevant de la consommation collective et ensuite voir si ces modifications auront comme résultat d'augmenter le nombre de femmes détenant des postes sur le plan municipal et régional. En élargissant les responsabilités des MRC, particulièrement avec le domaine des affaires sociales, ce palier devrait attirer plus de femmes.

Cette proposition est avancée, moins pour dire qu'elle doit représenter une priorité pour l'action politique des femmes que pour rappeler les liens qui existent entre les interprétations théoriques et les choix d'action. Si une augmentation importante dans les responsabilités municipales et régionales qui touchent à la consommation collective n'était pas suivie par une féminisation accrue du personnel politique, il faudrait alors revoir nos interprétations. 